\title{
Tangence
}

\section{Spirales et anneaux de Möbius dans Le processus et Le début de la fin de Marc-Antoine Mathieu Spirals and Möbius Strips in Le processus and Le début de la fin by Marc-Antoine Mathieu}

\section{Mélanie Lamarche-Amiot}

Numéro 68, hiver 2002

Littérature et mathématiques

URI : https://id.erudit.org/iderudit/008246ar

DOI : https://doi.org/10.7202/008246ar

Aller au sommaire du numéro

Éditeur(s)

Presses de l'Université du Québec

ISSN

0226-9554 (imprimé)

1710-0305 (numérique)

Découvrir la revue

Citer cet article

Lamarche-Amiot, M. (2002). Spirales et anneaux de Möbius dans Le processus et Le début de la fin de Marc-Antoine Mathieu. Tangence, (68), 33-49. https://doi.org/10.7202/008246ar
Résumé de l'article

Marc-Antoine Mathieu, graphiste de métier et bédéiste à ses heures, donne dans Le processus et Le début de la fin/La fin du début une direction plus qu'inhabituelle au fil qui conduit ses histoires. Il utilise les motifs de la spirale et de l'anneau de Möbius pour échafauder des labyrinthes narratifs complexes. Ces figures géométriques qui servent d'assises à l'intrigue sont repérables tant dans le texte que dans l'image et leur étude permet de saisir les subtilités d'une chronologie bouleversée par l'intrusion du rêve dans l'univers du héros. 


\title{
Spirales et anneaux de Möbius dans Le processus et Le début de la fin de Marc-Antoine Mathieu
}

\author{
Mélanie Lamarche-Amiot, Université Laval
}

\begin{abstract}
Marc-Antoine Mathieu, graphiste de métier et bédéiste à ses heures, donne dans Le processus et Le début de la fin/La fin du début une direction plus qu'inhabituelle au fil qui conduit ses histoires. II utilise les motifs de la spirale et de l'anneau de Möbius pour échafauder des labyrinthes narratifs complexes. Ces figures géométriques qui servent d'assises à l'intrigue sont repérables tant dans le texte que dans l'image et leur étude permet de saisir les subtilités d'une chronologie bouleversée par l'intrusion du rêve dans l'univers du héros.
\end{abstract}

Encore aujourd'hui, la bande dessinée est souvent perçue comme un art mineur, visant le délassement beaucoup plus que le travail formel. Ce jugement oublie toutefois que certains auteurs de bande dessinée, surtout depuis le renouvellement accompli dans les années 1960, ne se contentent pas de mettre une histoire en images (et en bulles), mais réfléchissent sur les possibilités offertes par le médium. En octobre 1992, Stanislas Barthélémy, Thierry Groensteen, Killofer, J.-C. Menu et Lewis Trondheim se réunissent pour fonder l'OuBaPo (l'Ouvroir de bande dessinée potentielle). La nouvelle cellule se greffe aux nombreux Ou-X-Po déjà existants et propose une démarche inspirée de celle de l'Oulipo. Des contraintes formelles tenant compte des composantes et des agencements propres à la bande dessinée ${ }^{1}$ sont créées au sein du groupe dont la composition varie peu au fil des années. Une étroite collaboration avec L'Association ${ }^{2}$ assure la diffusion régulière des projets d'auteurs. Bien que ne faisant pas partie des

1. Pour une présentation des travaux du groupe, voir $\mathrm{OuBaPo}$, Paris, L'Association, OuPus 1, janvier 1997.

2. Maison d'édition de bandes dessinées fondée en 1990 par David B, Killoffer, Lewis Trondheim, Mattt Konture, J.-C. Menu et Stanislas. 
membres en règle de l'OuBaPo, Marc-Antoine Mathieu publie quelques titres à L'Association et son travail dénote une inventivité s'inscrivant sans nul doute dans la visée de l'OuBaPo: de nombreux labyrinthes narratifs, temporels et picturaux lui permettent de remettre en question les codes et les normes du genre, non sans un usage très contrasté du noir et du blanc qui accentue l'effet de claustrophobie. L'origine, $L a Q u \ldots$.. Le processus et Le début de la fin, les quatre volumes de la série des Julius Corentin Acquefacques, prisonnier des rêves, témoignent d'une recherche formelle et esthétique originale et toujours ludique ${ }^{3}$.

Ces volumes, qui constituent autant de « discours sur la bande dessinée $[\ldots]$ placés sous le signe du rêve ${ }^{4}$ », mettent donc en scène un Julius Corentin Acquefacques évoluant dans des décors kafkaïens où rêve et réalité s'entremêlent sans cesse. Cette série recèle une abondance de procédés tant narratifs que picturaux qui contribuent à déjouer la linéarité traditionnelle du récit et à mettre en place des architectures textuelles et visuelles complexes, comparables à certaines figures géométriques. Avec le premier tome, L'origine, Mathieu instaure un climat d'inquiétante étrangeté en décloisonnant les frontières souvent imperméables qu'établissent aussi bien la fiction que la narration. Les courts-circuits qui en résultent s'apparentent ainsi à la notion de métalepse, si bien qu'on pourrait leur appliquer cette remarque de Gérard Genette: «Tous ces jeux manifestent par l'intensité de leurs effets l'importance de la limite qu'ils s'ingénient à franchir au mépris de la vraisemblance [...]; frontière mouvante mais sacrée entre deux mondes: celui où l'on raconte, celui que l'on raconte $^{5}$.» Le propos de L'origine est, en effet, la prise de conscience par le personnage de son statut de héros à deux dimensions dans une histoire ambiguë tramée par un auteur tout-puissant; dès lors, «l'histoire n'est plus seulement celle de l'homme Julius Corentin, personnage de bande dessinée, mais bien celle de Julius Corentin, personnage de bande dessinée confronté aux affres de son propre engendrement comme personnage dessiné ${ }^{6} »$. C’est sur cette vertigi-

3. Outre ces quatre bandes dessinées publiées chez Delcourt, Marc-Antoine Mathieu a fait paraître, à L'Association, La mutation, Le coeur des ombres et l'Ascension. Il a aussi participé aux collectifs Périphéries et Le retour de Dieu (ce dernier chez Autrement, collection «Histoires graphiques»). Deux nouveaux albums, Mémoire morte et Le dessin, ont paru chez Delcourt en 2000 et 2001.

4. Laurent Gerbier, "Les pièges de l'analogie », Neuvième Art, Angoulême, $\mathrm{n}^{\circ}$ 4, janvier 1999, p. 75.

5. Gérard Genette, Figures III, Paris, Seuil, coll. «Poétique», 1972, p. 245.

6. Laurent Gerbier, «Les pièges de l'analogie», art. cité, p. 76. 
neuse prise de conscience que toute la série repose et les mises en abyme comme les boucles temporelles étranges ${ }^{7}$ trouvent, en prenant appui sur une telle assise (borgésienne), un terrain propice à la multiplication des jeux possibles.

Le processus et Le début de la fin ont retenu notre attention parce que chacun d'eux module son récit autour d'une figure géométrique dissimulée dans le déroulement narratif ou diégétique des événements de l'album. Nous proposons donc une relecture de ces deux albums afin de mettre en évidence quelques procédés qui participent de la construction, en filigrane du dessin et de l'histoire, d'une spirale (Le processus) et d'un anneau de Möbius (Le début de la fin). Ces figures dictent le fonctionnement de l'ensemble de chacune des diégèses puisque ce sont elles qui produisent, lors de la lecture, l'effet d'étrangeté et de vertige caractéristique de la série des Julius Corentin Acquefacques, prisonnier des rêves.

Dans Le processus, un léger décalage temporel provoque la duplication à répétition du personnage de Julius Corentin. Le rêve et la réalité se côtoient dans un monde étrange où des médecins contrôlent l'inconscient de leurs patients en leur inoculant des rêves qui les guérissent de leurs angoisses nocturnes. Mais une erreur de traitement, causée par le dérèglement de l'horloge de Julius, lui fait vivre le rêve destiné à un autre patient. À partir de ce moment, Le processus entraîne le lecteur à travers une suite d'univers emboîtés débouchant les uns sur les autres dans la mouvance d'une réalité qui se dérobe sans cesse à elle-même. Dans Le début de la fin, ce sont les thèmes du reflet et du double, plutôt que celui du temps, qui déterminent les aventures de Julius dans le monde du rêve. Une torsion absurde du réel projette le personnage dans l'envers de son univers, dans un pur dédale onirique. Le personnage (dédoublé) évolue donc à contre-pied, emprisonné dans le double jeu d'un miroir qui ne reflète plus qu'une logique absurde digne du plus hallucinant des univers carrolliens. Ce dernier album de la série peint un univers réversible où les personnages évoluent simultanément sur l'envers et l'endroit d'un même ruban narratif. Si Julius tentait désespérément de freiner sa propre multiplication dans Le processus, il ne cherche plus, dans Le début de la fin, qu'à replacer chacune des représentations de son propre personnage dans l'espace qui lui convient.

7. La notion de «boucle étrange» provient de l'ouvrage de Douglas R. Hofstadter, Gödel, Escher, Bach. An Eternal Golden Braid, New York, Vintage , 1979. 


\section{Julius 1,2 et 3}

Le processus nous invite à suivre le parcours du personnage à travers trois univers en principe hermétiquement clos mais imbriqués les uns dans les autres. Le récit débute avec le réveil de Julius et se déroule à l'intérieur même de la Cité, jusqu'en son cœur où il découvrira l'Usine à rêves. C'est là que le premier changement de niveau de fiction apparaît, lorsque le personnage se met à rêver. Puis, à l'intérieur même du rêve, un second changement de niveau fictionnel survient de la rencontre avec le vortex. Julius, dans cette troisième portion de récit, ferme une boucle diégétique qui le ramène à son point de départ, c'est-à-dire dans son lit. Il ne peut plus cependant qu'être spectateur du recommencement (qu'on suppose infini) des aventures qu'il vient lui-même de vivre et dont il sera désormais exclu. Ainsi, Le processus met en scène un segment d'histoire qui présente le parcours complet effectué dans la diégèse par un personnage avant sa mise hors circuit. Le lecteur peut suivre toutes les étapes qui précèdent sa disqualification du parcours, celle-ci étant réalisée en même temps que la prise de conscience, par le personnage, de l'engrenage diabolique dans lequel il est coincé et dont nous étudierons plus en détail le fonctionnement.

Trois versions de Julius Corentin Acquefacques se côtoient dans Le processus. Un premier Julius apparaît au tout début, qui se lève, s'habille, se retourne et voit dans son lit un autre Julius, pareil à lui-même. Ce second personnage tente sans succès de dissuader le premier de se rendre à l'Usine de rêves, où il est convoqué. Suivi de près par le second, le premier Julius quitte malgré tout son appartement en direction de l'Usine. Parce que son horloge avance, cependant, Julius arrive trop tôt dans le bureau du docteur Koff. Ce décalage déclenche le mécanisme qui prendra Julius au piège: le savant et son équipe lui inoculent un rêve destiné au patient précédent sur la liste de rendez-vous. C'est à ce moment qu'apparaît un troisième Julius. Ce dernier n'est que le double du premier puisqu'il est issu de son rêve. L'introduction de ce troisième personnage, qui poursuivra la narration et la focalisation, ne provoque pas de coupure dans la trame narrative ni dans la mémoire du personnage. En fait, il s'agit ici d'un changement de niveau, du passage d'une réalité première à une réalité de degré supérieur d'abord présentée comme un rêve. La dernière case de la planche 25 (image 1) rassemble sur une même image, mais séparés par la frontière du rêve, trois états temporels différents du personnage qui correspondent aux trois étapes du processus. 


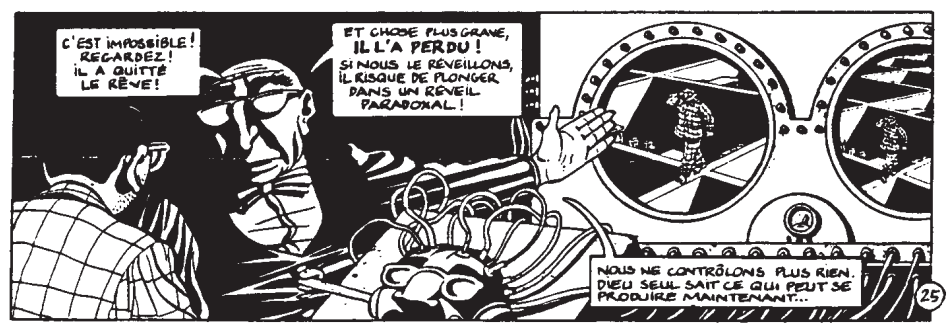

Image $1:$ Le processus, p. 27 , case du bas

Hérissé d'électrodes et profondément endormi, le premier Julius, dont on aperçoit seulement la tête au bas de l'image, a donné naissance à son double, qu'on voit en plus petit à travers le hublot de la machine. De dos, habillé d'un pyjama à carreaux, apparaît pour la dernière fois dans le récit le second Julius, celui qui est arrivé trop tard pour empêcher son double d'être branché sur la machine à rêves. Â cette étape du Processus, la multiplication effrénée des Julius ne permet pas encore d'anticiper la construction d'une spirale temporelle et narrative parfaite; la structure circulaire se devine toutefois à travers cette image qui constitue la dernière représentation du premier niveau de fiction dans l'album. En effet, un lecteur attentif notera peut-être que le Julius du rêve porte, contrairement au rêveur, un pyjama à carreaux identique à celui que porte le second Julius. Cet indice iconographique discret suggère la gémellité fonctionnelle des deux personnages, dont l'un est au début d'une chaîne d'actions que l'autre vient de compléter.

\section{De la fiction à la métafiction}

Le rêve est donc la voie permettant d'accéder à un nouveau niveau de fiction dans l'histoire. À partir de ce moment, en effet, le premier Julius dort et son double prend la relève de la narration et de la focalisation. Ce passage qui s'effectue en souplesse d'un niveau à l'autre est marqué, dans le dessin (image 2), par le passage d'une représentation en deux dimensions (comme sur l'image précédente) à une projection de cases en trois dimensions.

Julius, dans ce niveau de fiction, est conscient de s'être égaré dans le temps et cherche parmi les cases celle qui correspond à l'instant de son rêve. Chaque compartiment représente un moment, passé ou futur, de l'histoire. Le personnage se trouve, dans cette section de l'album qui correspond au deuxième niveau 


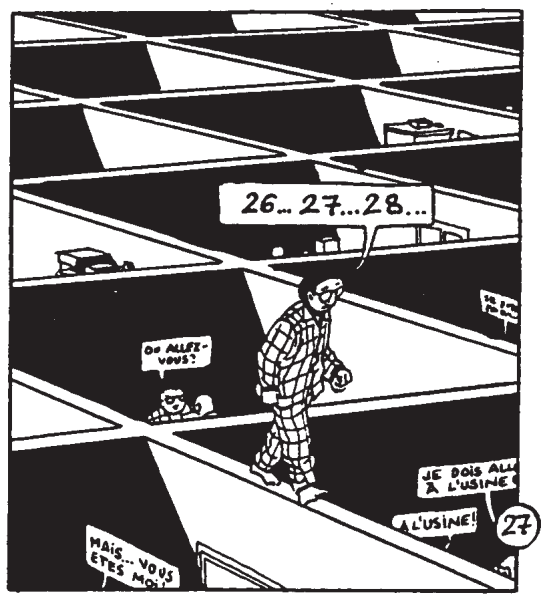

Image 2: Le processus, p. 29, case du bas à droite

de fiction, littéralement au-dessus du premier niveau et observe sa propre histoire en jetant un regard dans les cases qui se trouvent à ses pieds. Les cases du rêve évoquent du coup un édifice en damier dont on aurait enlevé le toit, motif qui provient peut-être du Diable boiteux de Lesage mais qui est ici réquisitionné à des fins nettement métafictionnelles. Il faut surtout noter que l'espace fictif parvient ainsi à intégrer le dispositif matériel de la bande dessinée tout en le soulignant. Le personnage, dans cet espace intermédiaire où il est à la fois dans la fiction et hors de celle-ci (puisqu'il peut contempler en lecteur, d'en haut, sa propre histoire), cherche la case qui correspond à son rêve pour la réintégrer: "L'immensité des cases renfermait toute mon histoire, classée comme dans un tiroir à une infinité de doubles-fonds. Ma vie n'était-elle donc qu'un rêve sans plafond? Ou une réalité sans fond? Bref... L'urgence me commandait pour le moment de me concentrer sur une seule réflexion: comment faire pour retrouver mon rêve ${ }^{8}$ ?...»

Quelques planches plus loin, Julius expérimente le vortex, «épicentre du rêve? foyer de la création? antre de l'absolu? cour du temps? spirale de la grande explication? trou blanc? avatar tridimensionnel? simple gag? ou tout cela à la fois ${ }^{9}$ ? », et est projeté dans le troisième et dernier niveau de fiction du récit. Le person-

8. Marc-Antoine Mathieu, Le processus, Bruxelles, Delcourt, 1993, planche 26.

9. Marc-Antoine Mathieu, Le processus, ouvr. cité, planche 31. 
nage, à la suite de cette rencontre, se retrouve hors du monde de la bande dessinée, parmi les pages de l'histoire étalées devant lui. La photo, dans cette troisième section de l'histoire, prend le relais du dessin. Pour accentuer ce point de tension que constitue le passage du deuxième au troisième niveau de fiction, Mathieu utilise, en plus de la photographie, le matériau même de la bande dessinée: le papier. En effet, ce changement de niveau est marqué par le déploiement d'une spirale réellement découpée dans l'album et qui relie la page 37 à la page 39 . Cet ingénieux procédé permet, de plus, de créer dans le monde réel du lecteur une représentation tangible de ce qu'est le vortex.

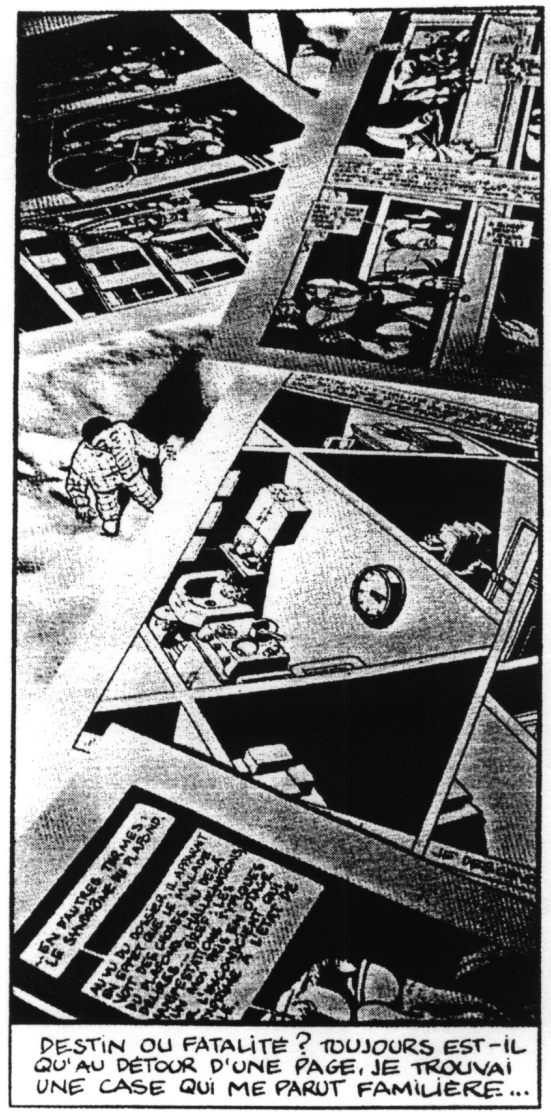

Image 3 : Le processus, p. 44, case du haut à gauche 
En se reconnaissant dessiné sur les planches éparses, Julius s'aperçoit que la suite de son histoire doit, elle aussi, se trouver dans ces pages; le personnage trouve effectivement une case (image 3) qui lui semble familière et qui, de plus, indique l'heure exacte à laquelle il se souvient avoir quitté son appartement. Il croit, dans un mouvement circulaire, revenir au premier niveau de fiction en réintégrant cette case. Cependant, au moment où il aperçoit un double de lui-même qui le regarde, éberlué, il comprend s'être trompé de rêve, mais il ne peut plus désormais s'échapper parce que le plafond, au-dessus de lui, a réapparu. Ce Julius, que nous avons suivi depuis le début de la narration, est devenu l'équivalent du second Julius des premières pages, celui qui tentait d'empêcher le personnage de se rendre à l'Usine de rêves. Le cycle, dès lors, recommence. Ce passage, d'un point de vue à l'autre, le dessin le symbolise à merveille.
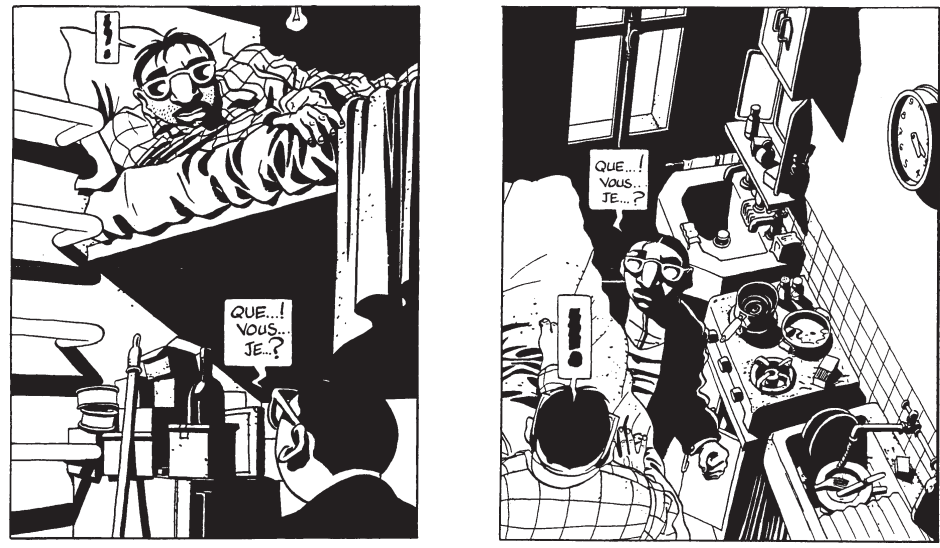

Images 4 et $5:$ Le processus, p. 8 , image du haut à gauche, et p. 46 , en haut à gauche

$\mathrm{Au}$ début du récit (image 4), le lecteur voit le second personnage à travers les yeux de Julius, de dos sur l'image. À la fin (image 5), la même situation se reproduit, mais la focalisation du personnage (et celle du lecteur) émane du point de vue opposé. Désormais, Julius est ce personnage anachronique qui se retrouve dans le lit.

La narration prise en charge par Julius se termine peu après, une fois qu'il a saisi le fonctionnement du processus. Il tente, comme son prédécesseur, de dissuader son double de se rendre à 
l'Usine. Pourtant, il reste une étape à accomplir avant que Julius n'ait effectué un parcours complet dans l'histoire. Julius devra partir à la poursuite de son double et le retrouver, malheureusement trop tard, branché à la machine à inoculer des rêves. Cette dernière étape dans le trajet du personnage, avant sa disqualification de l'histoire par le changement de niveau produit par le rêve, fait partie du processus; l'histoire comprend aussi cette tentative de stopper son recommencement infini. Cette dynamique autorégénératrice ne peut être interrompue puisque la tentative d'interruption est intégrée au processus lui-même, et c'est ce qui fait de l'histoire une spirale parfaite, inaltérable. Cette dynamique représente, en définitive, le mouvement de la diégèse et non celui du personnage. Elle se crée à partir de la combinaison du mouvement circulaire de l'histoire et de la translation verticale accomplie par les changements de niveau successifs. C'est au cours du premier changement de niveau (par l'inoculation du rêve) qu'est produit, à chaque cycle diégétique, un nouveau personnage permettant de poursuivre l'histoire et de la recommencer sans cesse.

Deux points de tension permettent donc les changements de niveaux: dans un premier temps, le rêve forcé de Julius, et dans un second temps, le passage par le vortex. Le premier changement de niveau permet d'isoler les personnages dans leurs univers respectifs; comme la communication entre les deux instances devient impossible, la poursuite du mécanisme est assurée. Le second changement de niveau mène, à la toute fin de l'album, à la réintégration d'un premier niveau de fiction, dans un cycle diégétique de degré supérieur; en réintégrant un univers dessiné, puis en se réveillant à nouveau dans sa chambre, Julius accomplit encore deux changements de niveau ${ }^{10}$. Cependant, comme ces changements ne le ramènent pas à son statut originel, on ne peut dire que la diégèse se replie sur elle-même. Malgré le retour à un point de départ événementiel identique à celui du début, il y a eu escalade dans le parcours du personnage: celui-ci ne répète pas les actions qu'il a déjà effectuées, mais se voit éjecté de l'histoire pour faire place à un double de lui-même produit par le processus, dans la machine à rêves. C'est donc bien une spirale (et non une simple boucle) qui régit le dispositif.

10. Les changements s'effectuent donc comme suit: diégèse (rêve) $\rightarrow$ niveau supérieur (vortex) $\rightarrow$ sortie des pages $\rightarrow$ niveau supérieur (réinsertion dans les planches) $\rightarrow$ diégèse (réveil). 


\section{Le début de la fin/La fin du début et l'anneau de Möbius}

La structure spiralée de l'histoire du Processus est rendue possible par les changements de niveaux narratifs associés au rêve. En effet, c'est par l'intermédiaire de la machine à rêves que le personnage se duplique, passe à un autre niveau de fiction et maintient, bien malgré lui, le mouvement circulaire de la structure diégétique. Dans Le début de la fin, c'est le motif du reflet qui sert de point de départ à une boucle temporelle étrange. Deux mondes possibles coexistent dans la fiction: le monde habituel, connu de Julius (et du lecteur familier de l'univers de Mathieu), et le monde plus étrange des reflets où tout est, comme il se doit, inversé: les personnages marchent sur les plafonds, les wagons cherchent en vain à entrer en collision sur les rails et les agents de police donnent de l'argent en guise de contravention. Julius s'éveille en faisant à l'envers les gestes de la vie quotidienne: il se rase et sa barbe pousse sous la lame, il marche à reculons et est désolé d'être en avance à son rendez-vous. On finira par comprendre que le Julius du monde des reflets s'est retrouvé par erreur parachuté dans le monde habituel de la fiction où le futur succède au passé. Mathieu entremêle ainsi des inversions de trois sortes dans Le début de la fin: spatiales, actantielles et temporelles.

Il n'y a pas de fin proprement dite à cet album concept qui renvoie son lecteur, à partir de deux débuts possibles, au centre de l'album. La première et la quatrième de couverture annoncent deux titres symétriques: Le début de la fin et La fin du début. La tranche affiche L'épaisseur du miroir. Au centre de la diégèse, le lecteur doit retourner l'album, dont les deux moitiés sont disposées tête-bêche, et reprendre à partir de la couverture opposée, sa lecture le ramenant au centre où les deux récits se rejoignent. Le début de la fin et La fin du début sont numérotés respectivement chapitres 1 à 5 et chapitres -1 à -5 . Cependant, ils ne sont pas, ni graphiquement ni du point de vue de l'histoire, l'exact reflet l'un de l'autre.

Dégager la figure de l'anneau de Möbius ${ }^{11}$ dans Le début de la fin relève davantage de l'interprétation que de la mise en relief d'un thème dominant, comme c'était le cas avec la spirale dans Le processus. L'emploi de l'anneau de Möbius comme figure structurante de l'album, bien que signalé par la présence effective d'un tel

11. Rappelons qu'un anneau de Möbius est une surface à un seul côté qui occupe les trois dimensions de l'espace et qui est obtenue en réunissant les deux extrémités d'un rectangle après avoir fait subir à l'une de ses extrémités une torsion de 180 degrés. 
anneau ${ }^{12}$, est plus discret que celui de la spirale dans Le processus, où celle-ci est constamment représentée. Pour saisir cette figure, nous devons nous représenter les personnages comme s'ils évoluaient sur une bande continue correspondant au déroulement chronologique de l'histoire. Le récit progresse sur ce ruban narratif; du fait de sa torsion, cependant, on peut aussi voir ce dernier comme une figuration du schéma actantiel, selon un parcours «tordu» qui traverse la frontière séparant le monde habituel de la fiction (l'endroit) de l'univers du rêve (où tout fonctionne à l'envers). Nous voyons donc que deux représentations du même personnage sont implicitement suggérées dans l'album: le Julius des premières cases et son double, le Julius qui doit en principe appartenir à l'univers des reflets. Or, on l'a vu, Le début de la fin met d'abord en scène un personnage qui se réveille dans un univers qui, en principe, est celui de son double. Ce réveil n'est toutefois qu'un faux indice puisque le lecteur apprend plus loin que le personnage est, depuis le début, en train de rêver. Selon le docteur Evariste Etsirave, en effet, l'absence du reflet de Julius dans le miroir signifie qu'il est le produit de son propre rêve, représentation de lui-même égarée dans un univers étranger.

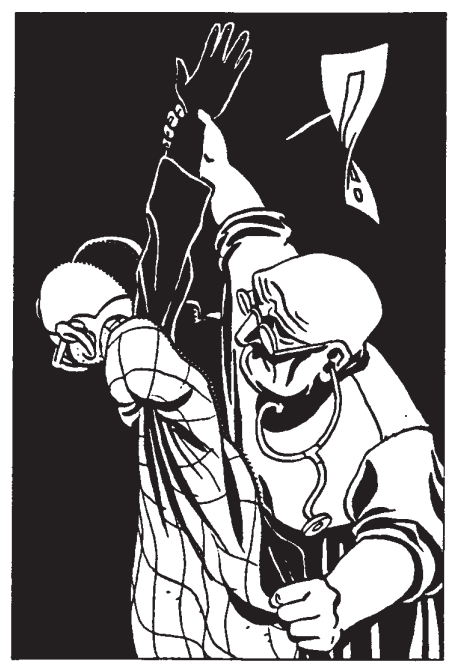

Image 6: Le début de la fin, planche 19, case du haut à gauche

12. Marc-Antoine Mathieu, Le début de la fin/La fin du début, Bruxelles, Delcourt, 1995, planche 11 . 
Pour remédier aux problèmes de Julius, le docteur Etsirave l'ouvre à l'aide d'une fermeture éclair (on s'aperçoit alors que le personnage est creux comme un vêtement) et le retourne comme un gant (image 6). Ainsi, il lui rend son reflet dans le miroir et il légitime la présence de Julius dans ce double monde de la fiction et du rêve, sans toutefois le départir de ses réactions propres à l'univers de l'envers. Cette opération délicate d'infraspatialisation ou "plus vulgairement: [de] retournement de situation ${ }^{13}$ ", constitue la première étape de la construction de l'anneau de Möbius dans le déroulement du Début de la fin. Il correspond à la demi-torsion nécessaire pour créer une figure géométrique à une seule surface. De plus, par cette opération, le docteur cristallise de manière définitive la position du personnage dans cet univers à deux faces: Julius retrouve son reflet dans le miroir et, selon la logique absurde de ce monde étrange, cela signifie qu'il en fait dès lors partie. L'illusion, en définitive, prend encore une fois le pas sur la réalité, bien relative, du prisonnier des rêves qu'est effectivement Julius Corentin Acquefacques.

\section{Du monde à l'anti-monde}

Au cour de l'album, dans la boutique d'un marchand de miroirs au détail nommé Speculum Muluceps, les personnages passent d'un univers à l'autre. Comme Alice, Julius traverse le miroir et se retrouve dans une réalité autre:

Je comprenais maintenant pourquoi l'ordre des choses était inversé. Je n'étais qu'un reflet, et je subissais les effets de l'inversion.

Là-haut, mon reflet se dirigeait à son tour vers le miroir, dans une symétrie logique.

Cette symétrie dont le miroir reflète le symbole.

Je réalisais que nous étions tous les deux destinés à rester aux abords du miroir... ou à revivre à l'envers ce qui s'était déjà passé.

Mais l'envers était-il absolument identique à l'endroit?

Un je-ne-sais-quoi m'en faisait irrésistiblement douter ${ }^{14}$.

Si l'on en juge par l'image qu'on peut voir sur les planches 26 et 27 (image 7), le reflet du personnage se dirige vers le miroir, de

13. Marc-Antoine Mathieu, Le début de la fin, ouvr. cité, planche 19.

14. Marc-Antoine Mathieu, Le début de la fin, ouvr. cité, planche 27. 

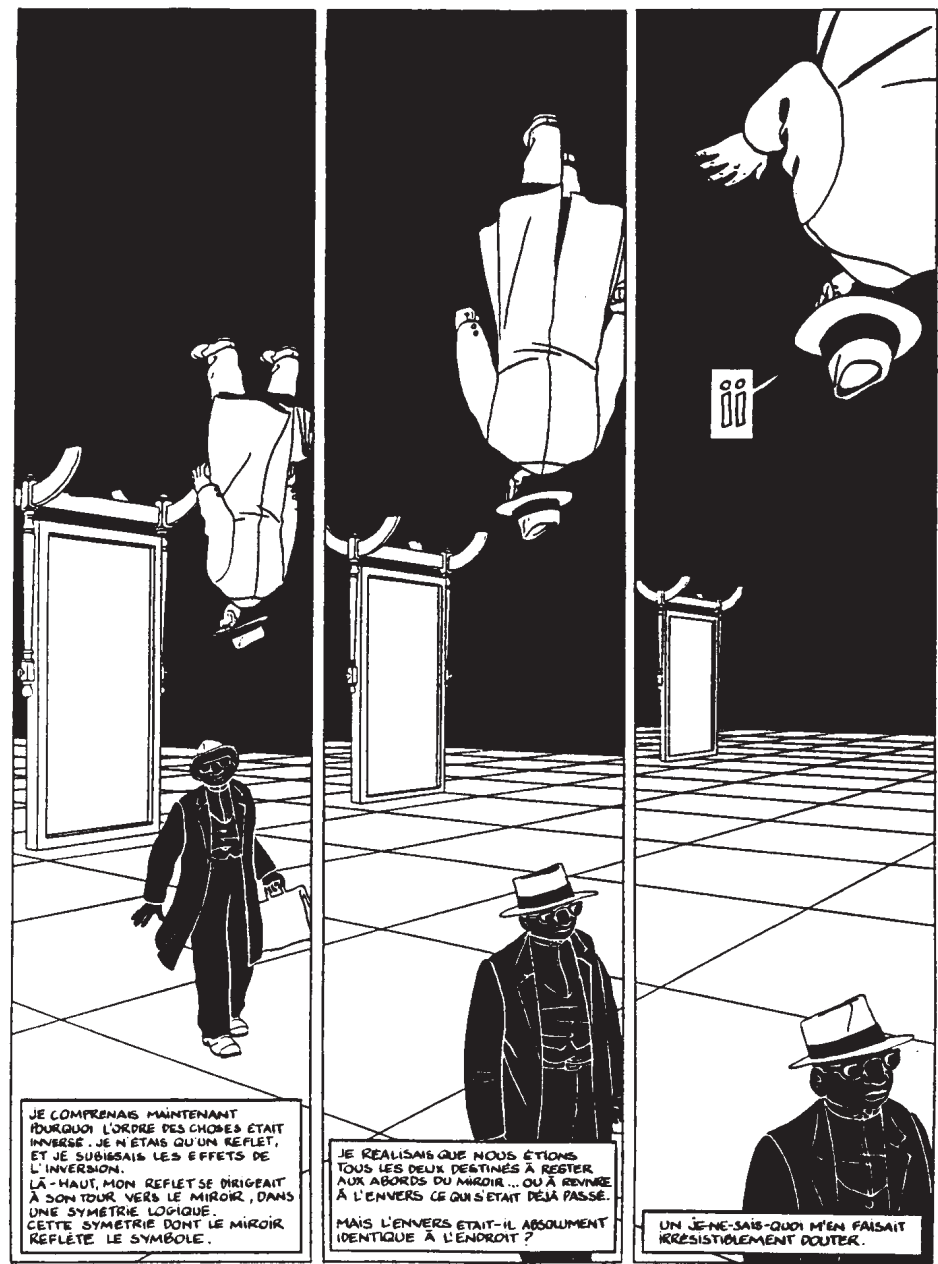

Image 7: Le début de la fin, pl. 27

face, au moment où Julius (devenu le personnage noir depuis son retournement) en sort. Aux planches 24 et 25, le personnage blanc, reflet de Julius, sortait du miroir au moment où celui-ci allait $y$ entrer. Il semblerait donc, si l'on observe la position des personnages, qu'il y ait non pas un seul axe de réflexion mais bien deux: l'axe horizontal et imaginaire, qui sépare le monde réel du monde du rêve, le personnage de son reflet, et l'axe vertical, représenté 
dans le livre par la psyché et sur la tranche, dans notre réalité, par le titre de la bande dessinée: L'épaisseur du miroir.

Partant de cette hypothèse, il est possible d'avancer que les personnages évoluent, de part et d'autre d'une surface qui séparerait les deux univers, en sens inverse. De chacune des deux extrémités du ruban, ils se rendent, face à face, vers son centre où se trouve le miroir de l'arrière-boutique de Speculum Muluceps. En traversant le miroir, le personnage passe du monde habituel au monde des reflets, où tout est inversé. Julius, cependant, revient à un mode d'agir habituel; du coup, il se trouve encore une fois en position contraire par rapport aux autres éléments de la fiction. C'est à ce moment, quand les personnages traversent le miroir, que se réalise la jonction entre les deux extrémités du ruban temporel qui sépare les univers. En effet, c'est au centre de la bande dessinée et au centre de la narration que la diégèse reprend, dans un mouvement circulaire. Quand le personnage traverse la psyché, le lecteur doit retourner le volume et recommencer sa lecture à partir de la face opposée, La fin $d u$ début (les deux parties étant, rappelons-le, reliées têtebêche). Un second cycle d'événements débute alors, qui répond à la première moitié de l'histoire en en reprenant, à quelques différences près (annoncées par les propos que Julius se tient à la planche 27), les principales actions.

Pour parcourir la surface complète d'un anneau de Möbius dans un univers en trois dimensions, il faut effectuer deux «cercles » dans l'espace: le premier correspond au parcours d'une face du ruban avant sa torsion et le second correspond, en réalité, au parcours de l'envers de cette première face. Il est toutefois impossible de distinguer en quel point l'une finit et l'autre commence puisqu'un anneau n'a ni début ni fin. Dans Le début de la fin/La fin $d u$ début, chaque section s'apparente aussi à une boucle (ouverte) puisque, au centre de la bande dessinée, le lecteur est ramené au début d'un parcours événementiel semblable à celui qu'il vient de parcourir dans la première partie de l'histoire. Pour compléter son parcours du ruban narratif, le lecteur doit effectuer une seconde boucle (image 8), qui correspond à l'envers de la première. L'anneau de Möbius se construit donc en réunissant, par le parcours de la lecture, les deux extrémités d'un ruban imaginaire qui symbolise à la fois le déroulement chronologique de l'histoire et l'axe de symétrie horizontal séparant les deux univers de la fiction. Les personnages passent, en traversant la psyché de Spéculum Muluceps, d'un monde à l'autre. En admettant l'hypothèse que la lecture du Début de la fin suive un schéma circulaire, il est possible de soute- 
nir que cette forme ne comporte qu'une seule surface, puisque les personnages s'y déplacent sans changer de niveau de fiction. Le lecteur suit ainsi le Julius du monde des rêves dans la portion de l'histoire qui correspond au monde de l'endroit; puis, après le passage à travers le miroir, il retrouve le personnage "habituel» dans le monde des reflets (des rêves) jusqu'à la seconde traversée du miroir le ramenant au point de départ. Chaque segment pourrait donc représenter un cercle dans la géométrie imaginaire à trois dimensions, lequel permet la représentation de l'anneau de Möbius. Bref, une moitié de l'album correspond à la fois à l'un des deux mondes parallèles et au parcours de la moitié de la surface de l'anneau.

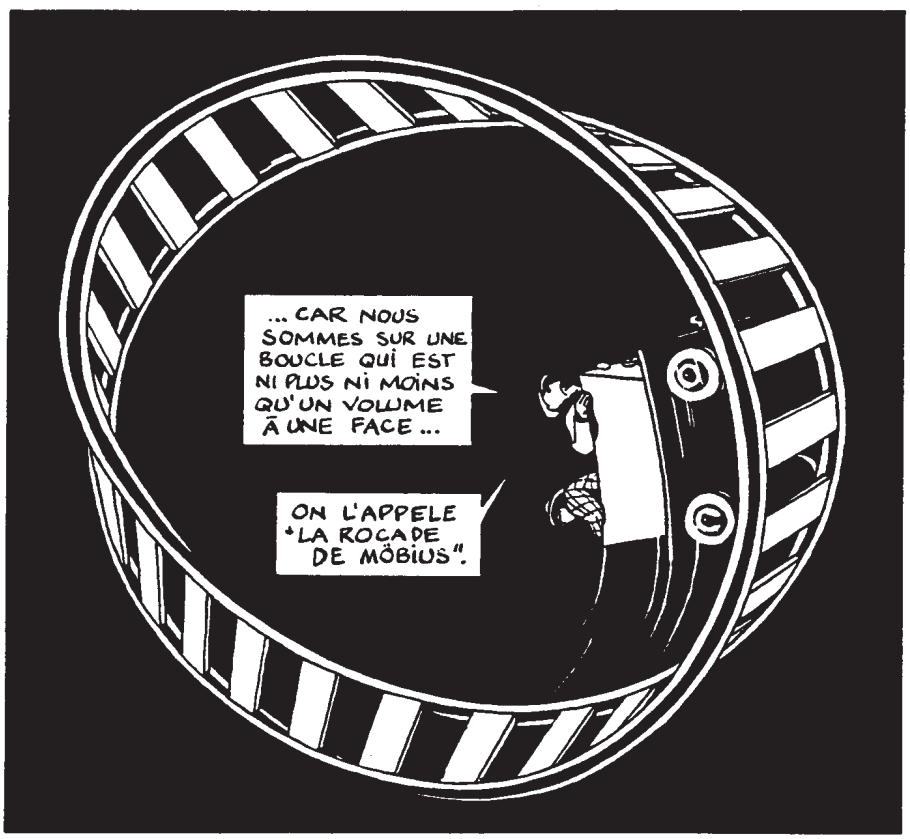

Image 8: Le début de la fin, pl. 11, en haut à droite

Bien que les deux segments de l'histoire ne se répondent pas parfaitement, ils partent de points semblables (les rêves de pile ou face) et convergent vers un nœud unique (le miroir central). Le motif du reflet, comme celui de la spirale dans Le processus, est maintes fois représenté dans le dessin et par le parcours de la 
diégèse. L'usage récurrent du palindrome, entre autres, renforce cette insistance thématique. Les noms de presque tous les personnages, sauf celui de Julius, se composent de deux termes identiques inversés: Speculum Muluceps, Evariste Etsirave, Urbain Niabru, etc. Les numéros de téléphone sont, eux aussi, soumis aux mêmes lois: 53835. Notons que le terme central, qui sert d'axe de réflexion aux autres termes, le chiffre 8 , est aussi une structure symétrique double. Un premier axe de symétrie sépare les deux cercles qui forment le 8 et un axe vertical peut tout aussi bien se dessiner au centre du 8 pour en refléter les moitiés gauche et droite. De plus, si l'on fait subir une rotation de 90 degrés au chiffre 8 , il devient le symbole de l'infini $(\infty)$. Cette astuce se remarque aussi dans le numéro de l'arrêt du wagonnet qui sert au transport en commun: 1203021. Le terme central, le 3, ne possède qu'un axe de symétrie horizontal. Cependant, le chiffre 3 peut tout aussi bien représenter l'une des moitiés verticales du 8 , son reflet étant, comme celui de Julius, absent. Dans la première partie, Le début de la fin, ce wagonnet se déplace sur la ligne 16 et dans la seconde partie, La fin du début, le 16 devient 19.

Ces observations mènent à une interrogation plus vaste sur la structure même de l'histoire. Il semble en effet possible d'avancer que le thème du reflet s'articule selon deux axes, l'un vertical et l'autre horizontal. Ceci expliquerait l'image centrale du livre qui nous montre les personnages traversant, face à face, le miroir de Speculum Muluceps. Mais n'est-il pas tout aussi pertinent d'avancer qu'il y aurait non pas un seul personnage et son reflet, mais bien deux personnages semblables et leurs reflets? Du fait que les deux parties peuvent être lues dans n'importe quel ordre et qu'elles sont données comme le reflet l'une de l'autre, il serait plausible de croire que les deux Julius s'avancent en même temps vers le miroir central, avec chacun son propre reflet au-dessus ou au-dessous de lui.

D'aucuns ont reproché au Début de la fin/La fin du début de céder à la tentation du formalisme. Pourtant, loin de pouvoir être entièrement démystifié par une lecture attentive de ses mécanismes, cet album dissimule, sous de nombreux procédés formels rigides, un réseau effervescent d'énigmes qui ne sauraient être résolues sans difficulté. Mathieu semble en effet s'être amusé à truffer son travail de quelques erreurs que l'on peut soupçonner être délibérées, reprenant ainsi l'idée oulipienne de clinamen. Ceci expliquerait en partie que les deux sections se répondent sans se refléter parfaitement. À l'intérieur même de l'album, des détails 
brisent aussi la symétrie attendue. Par exemple, le nom du souslocataire de Julius, Norbert Trébon, n'est pas un palindrome parfait. La barque dans laquelle Julius prend place au début de chacune des sections présente une différence d'importance: l'une est baptisée Le choix, tandis que l'autre porte un triangle vide en guise d'enseigne. Que ce soit au point de vue de la forme, du dessin ou de l'histoire, les bandes dessinées de Marc-Antoine Mathieu recèlent nombre de pièges de lecture qui ne peuvent être tous "désamorcés » dans le cas d'une étude aussi brève.

Le processus s'intègre parfaitement à la tentative de dégager de l'histoire ou du récit la construction d'une figure géométrique, en l'occurrence la spirale. L'anneau de Möbius dans Le début de la fin/La fin du début apparaît davantage comme une lecture possible que comme un thème incontournable. Ceci s'explique sans doute par le fait que la figure de la spirale, dans Le processus, est inscrite dans la structure narrative de l'album: la diégèse complète repose sur une spirale inaltérable qui devient le propos central de l'album. L'anneau de Möbius, dans Le début de la fin/La fin du début, s'impose plutôt comme un effet de lecture, l'un des multiples sens possibles que décèle après coup un lecteur curieux. 